\title{
MR-targeted TRUS prostate biopsy using local reference augmentation: initial experience
}

\author{
Wendy J. M. van de Ven ${ }^{1}$ Wulphert Venderink ${ }^{1} \cdot$ J. P. Michiel Sedelaar ${ }^{2} \cdot$ \\ Jeroen Veltman ${ }^{3} \cdot$ Jelle O. Barentsz $^{1} \cdot$ Jurgen J. Fütterer $^{1} \cdot$ Erik B. Cornel $^{4}$. \\ Henkjan J. Huisman ${ }^{1}$
}

Received: 30 January 2016 / Accepted: 28 March 2016 / Published online: 11 April 2016

(C) The Author(s) 2016. This article is published with open access at Springerlink.com

\begin{abstract}
Purpose To evaluate MR-targeted TRUS prostate biopsy using a novel local reference augmentation method.

Patients and methods Tracker-based MR-TRUS fusion was applied using local reference augmentation. In contrast to conventional whole gland fusion, local reference augmentation focuses the highest registration accuracy to the region surrounding the lesion to be biopsied. Pre-acquired multi-parametric MR images (mpMRI) were evaluated using PIRADS classification. T2-weighted MR images were imported on an ultrasound machine to allow for MRTRUS fusion. Biopsies were targeted to the most suspicious lesion area identified on mpMRI. Each target was biopsied 1-5 times. For each biopsied lesion the diameter, PIRADS and Gleason scores, visibility during fusion, and representativeness were recorded.

Results Included were 23 consecutive patients with 25 MR suspicious lesions, of which 11 patients had a previous negative TRUS-guided biopsy and 12 were biopsy naïve. The cancer detection rate was $64 \%$ (Gleason score $\geq 6$ ). Biopsy was negative (i.e., no Gleason score) in seven patients confirmed by follow-up in all of them (up to 18 months). After MR-TRUS fusion, $88 \%$ of the lesions could be visualized on TRUS. The cancer detection
\end{abstract}

Wendy J. M. van de Ven

Wendy.vandeVen@radboudumc.nl

1 Department of Radiology and Nuclear Medicine, Radboud University Medical Center, P.O. Box 9101, 6500 HB Nijmegen, The Netherlands

2 Department of Urology, Radboud University Medical Center, Nijmegen, The Netherlands

3 Department of Radiology, ZGT, Hengelo, The Netherlands

4 Department of Urology, ZGT, Hengelo, The Netherlands rate increases with increasing lesion size, being $73 \%$ for lesions larger than $10 \mathrm{~mm}$.

Conclusion Tracker-based MR-TRUS fusion biopsy with local reference augmentation is feasible, especially for lesions with an MR maximum diameter of at least $10 \mathrm{~mm}$ or PIRADS 5 lesions. If this is not the case, we recommend in-bore MR-guided biopsy.

Keywords MR-US fusion · Prostate cancer · Biopsy · PIRADS

\section{Introduction}

An elevated or rising PSA followed by systematic (on average 12 core) transrectal ultrasound (TRUS)-guided biopsy (USgBx) is the currently internationally accepted diagnostic procedure to detect prostate cancer and determine patient management [1]. TRUS cannot localize malignant tissue and is merely used to guide systematic biopsies. USgBx has a low sensitivity (40\%) [2-4], causing three problems: (1) Significant cancers can be missed or underestimated; (2) there is unnecessary overtreatment due to overdiagnosis [5-7]; and (3) it may lead to repeat biopsies inducing increased infection rates [8]. Therefore, multiparametric MR imaging and MR-guided biopsy might be a better alternative.

Multi-parametric MR imaging (mpMRI) has recently emerged as a diagnostic technique that can accurately localize significant cancer in the prostate [9, 10]. In-bore MR-guided MR biopsy (MRgMRBx) has been shown to (1) reduce the detection of low-risk cancer and (2) increase the detection rate of intermediate- and high-risk cancer, while using fewer cores [11]. However, the associated cost, relative complexity, and inconvenience of MRgMRBx may 
prevent widespread adaption in clinical practice. An alternative biopsy method for MR-guided biopsy would be welcome.

MR-guided TRUS fusion biopsy (MRgUSBx) has recently emerged [12-14]. This allows to combine the high accuracy of mpMRI with the ease and accessibility of TRUS. However, for $95 \%$ correct Gleason grading, a 1.9$\mathrm{mm}$ accurate spatial registration of MR and US is required [15]. Most MRgUSBx devices do not achieve this accuracy in practice $(3-6 \mathrm{~mm}[12,16])$. Accuracy can be slightly increased by taking one or more additional cores [17].

Two MRgUSBx strategies can be distinguished: cognitive and computational fusion. The fastest and simplest form of computational fusion is tracker-based rigid registration, using an electromagnetic (EM)-tracker [18, 19]. An EM-tracker attached to a TRUS probe tracks its position and orientation allowing to link a live TRUS image to a prerecorded MR image. We previously performed a phantom study on EM-tracker registration and estimated the registration accuracy in 3D to be $5-7 \mathrm{~mm}$ [20]. Current rigid MR-TRUS fusion protocols focus on optimizing accuracy for the entire gland volume. Due to prostate deformation, the registration accuracy can never be optimal within the whole gland. We hypothesize that by restricting the registration to the partial gland volume surrounding the lesion, a more consistent and possibly better registration accuracy can be achieved within this partial volume containing the lesion. The EM-tracker approach we use in our study allows to do this quickly, which, combined with visual feedback, can lead in a few iterations to an augmented, focal match of TRUS and MR imaging. We propose a novel protocol to augment the accuracy locally by selecting reference landmarks on both MR and TRUS images that are close to the biopsy target [21], which we refer to as local reference augmentation in analogy to all-weather aircraft landing systems.

The aim of this study is to evaluate our novel EM-tracker MR-TRUS fusion biopsy protocol using local reference augmentation in regular clinical practice. To our knowledge, this is the first report on a locally optimized MRTRUS fusion biopsy method. We will explore the capability of sampling mpMRI suspicious lesions and get insight into the representativeness of the biopsy result. Additionally, we will determine the proportion of tumors confidently visible on TRUS after fusion.

\section{Patients and methods}

\section{Patient population}

Inclusion criteria for our study were patients scheduled for MRgUSBx who had an mpMRI showing a lesion scored as PIRADS $\geq 4$ or PIRADS 3 with additional clinical suspicion (e.g., unusually high PSA, persistent rising PSA). Biopsy was performed at the Radboudumc (Nijmegen, the Netherlands) or at the ZGT (Hengelo, the Netherlands). The study was approved by the Institutional Review Board of the Radboudumc for MR lesions $>9 \mathrm{~mm}$, and all included Radboudumc patients gave their written informed consent. The requirement to obtain institutional review board approval was waived at ZGT as MRgUSBx was their regular clinical procedure in prostate cancer diagnosis. Our data set contains all patients included at the Radboudumc and ZGT between September 2013 and October 2014.

\section{Multi-parametric magnetic resonance imaging}

Prostate imaging mpMRI sequences were compliant to the ESUR guidelines [22] and included three orthogonal T2-weighted, diffusion-weighted, and dynamic contrastenhanced (DCE) series. Apparent diffusion coefficient (ADC) maps were calculated by the scanner. DCE used a gadolinium-based contrast agent by injecting $15 \mathrm{~mL}$ of Dotarem intravenously. Preferentially, we also added a 3D T2-weighted sequence with an isotropic resolution of $1 \mathrm{~mm}$ for MRgUSBx. Images of the entire prostate gland and seminal vesicles were obtained using a 3 Tesla MRI scanner (MAGNETOM Trio or Skyra; Siemens, Erlangen, Germany) with either a pelvic phased-array coil or a combination of an endorectal and pelvic phased-array coil.

Several genitourinary radiologists experienced in prostate MRI prospectively evaluated the mpMRI in a regular clinical setting, using structured reporting with the ESURstandardized PIRADS classification [22]. The location of each lesion was stored on an in-house-developed mpMRI analysis, viewing and reporting workstation (ProCAD) [23]. All mpMRI evaluations were discussed in a consensus meeting and adapted if necessary.

\section{Biopsy procedure}

An Aplio 500 (Toshiba Medical Systems, Japan) ultrasound device with an end-firing transrectal transducer (PVT781VT; Toshiba Medical Systems, Japan) was used for the MRgUSBx. Previously obtained T2-weighted MR images were uploaded to the ultrasound device. The original mpMRI including PIRADS scores were displayed on our mpMRI workstation, available during the fusion procedure. For a peripheral zone lesion, the biopsy target was the darkest lesion region on ADC; for the transition zone, it was the most suspicious area on the T2-weighted series. The MR target location was first identified on the workstation displaying the mpMRI and then re-located on the uploaded T2-weighted image. 


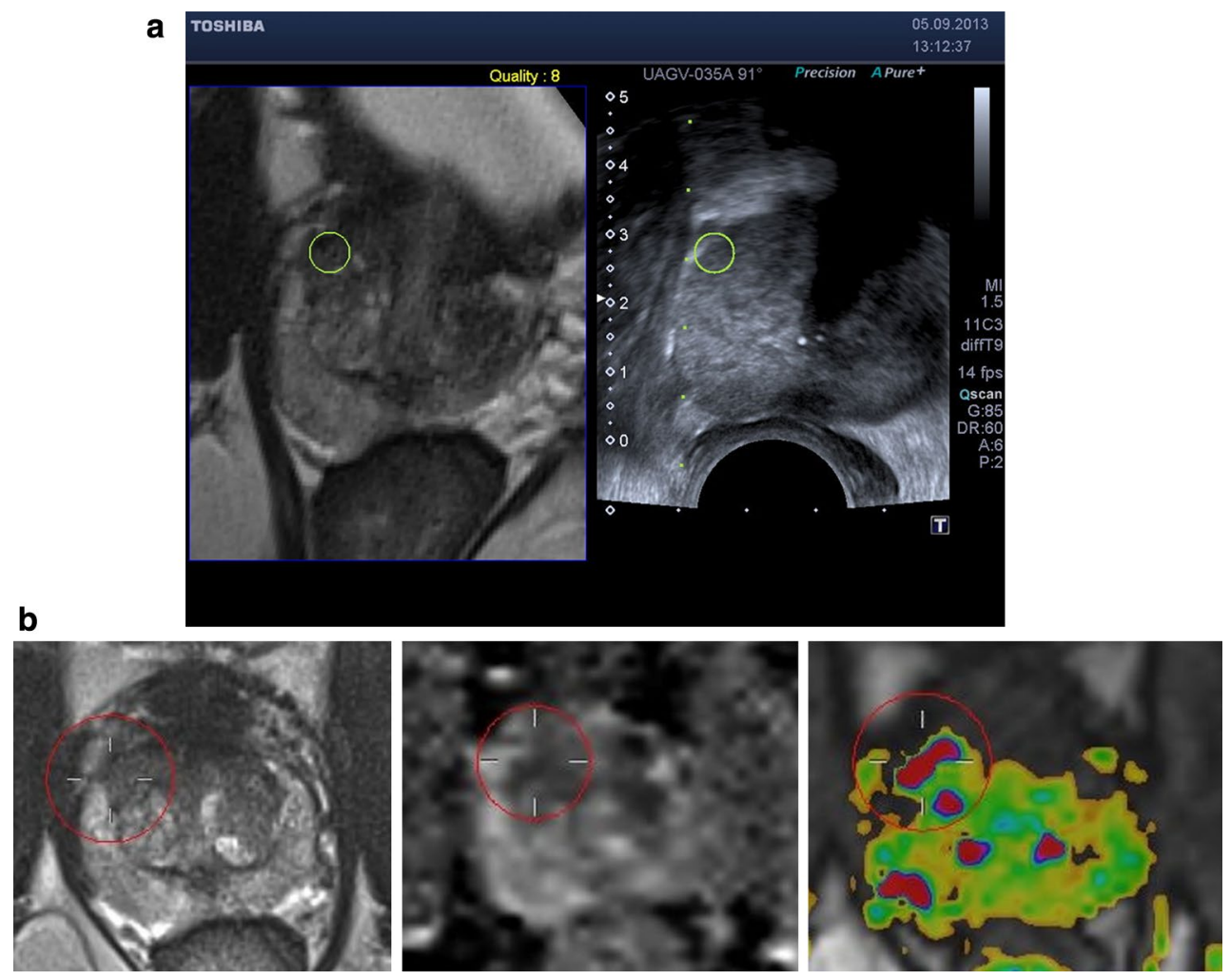

Fig. 1 a Screenshot of the Toshiba Aplio 500 during MRgUSBx. The green circle indicates the target as reported on mpMRI, projected on the US image after fusion. The dotted green line indicates trajectory along which the needle will shoot in the prostate (to be moved

Patients were positioned in the left lateral position for biopsy, similar to USgBx. The TRUS probe was inserted rectally with gel. A needle guide was placed onto the transducer. The ultrasound machine had a SmartFusion option that includes an EM position sensor attached to the TRUS transducer to spatially correlate imported 3D MR images and US in real time. The SmartFusion EM-tracker-based fusion is a two-step process. First, the US scanning orientation is matched to a variable MR image-reformatted orientation by manually selecting the best matching reformatting angle. Secondly, the correct anatomical 3D position is linked by selecting the same reference anatomical landmark in both images. During the biopsy procedure, the live US and the pre-acquired transversal T2-weighted images were shown simultaneously, allowing MR image guidance (example shown in Fig. 1). The accuracy of the EM-based method was enhanced by our novel local reference augmentation, i.e., the reference landmark used for synchronizing $3 \mathrm{D}$ position was selected close to the target location as identified on MR (example shown in Fig. 2). slightly for correct targeting in this screenshot). b The corresponding mpMRI with from left to right the transversal T2-weighted image, ADC map, and DCE image. These images were displayed using Pro$\mathrm{CAD}$ and were available during the fusion procedure

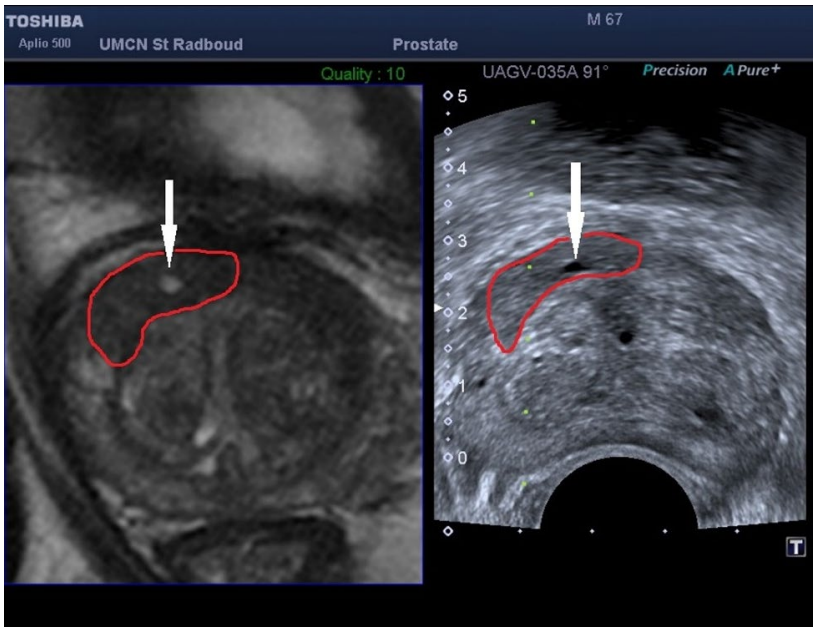

Fig. 2 Screenshot of the Toshiba Aplio 500 demonstrating the use of anatomical landmarks used for local reference augmentation. Reference landmarks are (iteratively) selected close to the target location. This example shows a cyst (indicated by the white arrow) inside the lesion (segmented in red) 
The MR-identified lesion location is then re-located on ultrasound using the fused image display. Visible mismatch was minimized by repeating the landmark selection, compensating for landmark localization errors or patient movement. In case the lesion was visible on TRUS after initial fusion, the biopsy was targeted to the TRUS location. Note that visibility in this respect means that lesions become visible on ultrasound only during the MR targeting fusion procedure. They are much less visible on ultrasound as such without the aid of fusion. Each mpMRI-detected target was biopsied 1-5 times. MR-TRUS fusion screenshots were stored during the biopsy to record the exact needle core location as part of the procedure to assess the representativeness.

\section{Histopathology}

Similar to all prostate biopsy procedures, all biopsy core specimens were examined by one of two specialized urogenital pathologists and graded according to the 2005 International Society of Urological Pathology Modified Gleason Grading System [24]. For stratification of biopsy data into significant and insignificant cancer, we applied the criteria for MRgMRBx as published by Pokorny et al. [11]. In short, lesions with a Gleason score $\geq 7$ in at least one of the MRgUSBx cores were defined as being clinically significant, as well as high-volume Gleason $3+3$ (i.e., tumor length $>6 \mathrm{~mm}$ or more than 2 positive cores). Low-volume Gleason $3+3$ or Gleason scores $<6$ were considered clinically insignificant, and lesions for which no Gleason score could be determined were considered negative. The cancer detection rate was based on lesions with Gleason score $\geq 6$. The pathology results were correlated with the MR-TRUS fusion biopsy images and the original mpMRI study.

\section{Biopsy evaluation}

A urologist in consultation with a radiologist evaluated the pathological outcome of the biopsy, taking the PIRADS scores of the mpMRI into consideration. In case the pathological outcome is lower than what would be expected based on mpMRI, the patient would need a re-biopsy. The biopsy was considered representative if the patient was not scheduled for an immediate MRgMRBx re-biopsy. As part of the radiological quality control procedure, all mpMRI studies that did not have significant cancer after MRgUSBx were reevaluated by two expert prostate radiologists in consensus. Furthermore, follow-up results of patients were collected.

\section{Data analysis}

As an indication of feasibility, the cancer detection rate and number of non-representative biopsies were analyzed.
Earlier research indicated that PIRADS and Gleason score, as well as visibility and lesion size, had an effect upon the detection rate, and therefore we also performed subgroup analyses. Statistical proportion analysis was performed to determine the cancer detection rate for each (sub)group. Three different size groups were created $(0-10,11-20$, and $\geq 20 \mathrm{~mm})$. Finally, the mean number of cores taken per lesion was determined for each size group.

\section{Results}

Between September 2013 and October 2014, 23 consecutive patients with 25 mpMRI suspicious lesions underwent MRgUSBx and were included (two patients had two lesions). Table 1 summarizes the general characteristics of the included patients. Eleven patients had at least one previous negative TRUS biopsy session, one patient had a previous negative MRgMRBx 1 week before MRgUSBx (but representativeness of MRgMRBx was uncertain), and the other 11 patients were biopsy naïve. The prospective mpMRI scores were: 3 PIRADS 3 lesions, 9 PIRADS 4 lesions, and 13 PIRADS 5 lesions. Most of the mpMRI suspicious lesions were located in the peripheral zone (20/25).

All MRgUSBx were considered representative, none needed an immediate re-biopsy. Table 2 shows the results

Table 1 Summary of patient characteristics

\begin{tabular}{llllc}
\hline Parameter & \multicolumn{4}{l}{ All patients, $n=23$} \\
\cline { 2 - 5 } & Mean & SD & Median & Range \\
\hline Age (years) & 63 & 6.4 & 65 & $51-75$ \\
PSA (ng/mL) & 10.3 & 6.2 & 8.9 & $2.9-29.3$ \\
\hline
\end{tabular}

Table 2 Results of prostate biopsies

\begin{tabular}{ll}
\hline Parameter & Value \\
\hline No. of patients/lesions & $23 / 25$ \\
No. of patients/lesions with cancer (GS $\geq 6$ ) & $16 / 16$ \\
Total no. of cores & 64 \\
No. of positive cores (GS $\geq 6$ ) & 28 \\
Mean primary Gleason grade & $3.19 \pm 0.39$ \\
Mean secondary Gleason grade & $3.25 \pm 0.43$ \\
Mean Gleason score (GS) & $6.43 \pm 0.50$ \\
No. of GS 3+3 & 9 (of which 4 were \\
& clinically signifi- \\
& cant) \\
No. of GS 3+4 & 4 \\
No. of GS $4+3$ & 3 \\
\hline
\end{tabular}


Table 3 Cancer detection rates per PIRADS score and TRUS lesion visibility, including $95 \%$ confidence intervals (CI)

\begin{tabular}{lcllcc}
\hline Category & $\begin{array}{l}\text { No. } \\
\text { of lesions }\end{array}$ & $\begin{array}{l}\text { No. of lesions } \\
\text { with any cancer }\end{array}$ & $\begin{array}{l}\text { Proportion } \\
(95 \% \text { CI }) \text { any cancer }\end{array}$ & $\begin{array}{l}\text { No. of lesions } \\
\text { with significant cancer }\end{array}$ & $\begin{array}{l}\text { Proportion (95 \% CI) } \\
\text { significant cancer }\end{array}$ \\
\hline PIRADS 3 & 3 & 2 & $67 \%(20-94 \%)$ & 2 & $67 \%(20-94 \%)$ \\
PIRADS 4 & 9 & 4 & $44 \%(19-73 \%)$ & 3 & $33 \%(12-65 \%)$ \\
PIRADS 5 & 13 & 10 & $77 \%(49-93 \%)$ & 6 & $46 \%(23-71 \%)$ \\
TRUS visible & 22 & 14 & $64 \%(43-80 \%)$ & 10 & $45 \%(27-65 \%)$ \\
TRUS invisible & 3 & 2 & $67 \%(20-94 \%)$ & 1 & $33 \%(6-80 \%)$ \\
All & 25 & 16 & $64 \%(44-80 \%)$ & 11 & $44 \%(27-68 \%)$ \\
\hline
\end{tabular}

of the prostate biopsies. In summary, the median number of targeted cores taken per lesion was 2 (range 1-5). Cancer (Gleason score $\geq 6$ ) was detected in 16 of the 25 lesions $(64 \%)$ and 16 of 23 patients $(70 \%)$. Two patients had a second lesion with Gleason score $\geq 6$. MRgUSBx was negative in 7 patients: 2 had a PIRADS 5 lesion, 4 had a PIRADS 4 lesion, and 1 had a PIRADS 3 lesion. Patients with negative MRgUSBx were referred to active surveillance based on PSA or follow-up mpMRI after 3-6 months.

The cancer detection rates per PIRADS score are shown in Table 3. The cancer detection rate of the PIRADS 5 lesions was $77 \%$, of PIRADS 4 lesions $44 \%$, and of the PIRADS 3 lesions $67 \%$. Pathological biopsy outcomes per PIRADS score are shown in Fig. 3a. Clinically significant lesions were present in 46, 33, and $67 \%$ of the PIRADS 5, 4 , and 3 lesions, respectively.

During the biopsy procedure, 23 of the lesions (88\%) could be visualized on TRUS after image registration. For TRUS visible lesions, biopsies were targeted to the location as visible on TRUS. The cancer detection rate of the TRUS visible lesions was $64 \%$ and that of the TRUS invisible lesions $67 \%$ (see Table 3). For clinically significant cancer, this changes to 45 and $33 \%$ for TRUS visible and invisible lesions, respectively. The pathological outcomes for TRUS visibility are shown in Fig. 3 b.

In Table 4 the lesions are grouped according to their size. The cancer detection rate is higher for the larger lesions, on both lesion and core basis. For lesions larger than $10 \mathrm{~mm}$, the cancer detection rate is $73 \%$ (Gleason score $\geq 6$ ). Also, slightly more cores are taken for larger lesions.

The follow-up of the mpMRI suspicious patients with a negative or insignificant MRgUSBx outcome was collected, and results are shown in Table 5. In summary, follow-up results showed that 3 of 7 patients with negative MRgUSBx had stable or decreasing PSA after 6-18 months and one patient is in active surveillance. For the remaining three, one had negative USgBx after
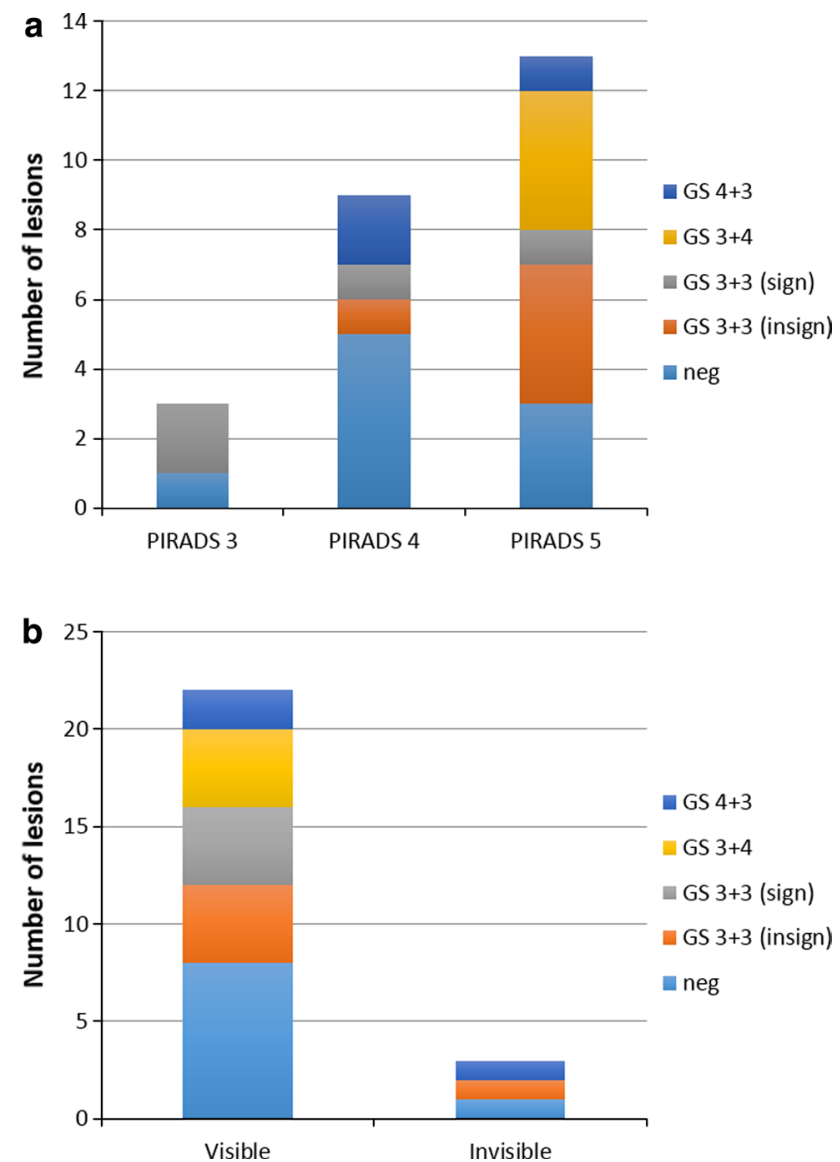

Fig. 3 The number of lesions detected with targeted MRgUSBx according to $\mathbf{a}$ the PIRADS score on mpMRI and $\mathbf{b}$ the visibility of the lesion on TRUS

12 months, one had negative MRgMRBx after 12 months, and one showed a PIRADS 2 lesion on follow-up mpMRI. For the five patients with insignificant MRgUSBx, one patient was lost to follow-up, one patient has decreasing PSA, one patient is scheduled for biopsy, and two are still in active surveillance. 
Table 4 Biopsy outcomes grouped according to lesion size on mpMRI

\begin{tabular}{lllll}
\hline $\begin{array}{l}\text { Largest } \\
\text { diameter }(\mathrm{mm})\end{array}$ & $\begin{array}{l}\text { No. } \\
\text { of lesions }\end{array}$ & $\begin{array}{l}\text { Mean no. } \\
\text { of cores (range) }\end{array}$ & $\begin{array}{l}\text { Cancer (GS } \geq 6) \\
\text { detection rate per lesion (95 \% CI) }\end{array}$ & $\begin{array}{l}\text { Cancer (GS } \geq 6) \\
\text { detection rate per core (95 \% CI) }\end{array}$ \\
\hline $0-10$ & 10 & $2.4(2-3)$ & $50 \%(24-76 \%)$ & $33 \%(18-53 \%)$ \\
$11-20$ & 11 & $2.5(1-5)$ & $64 \%(35-85 \%)$ & $44 \%(28-63 \%)$ \\
$>20$ & 4 & $2.8(2-4)$ & $100 \%(45-100 \%)$ & $73 \%(43-91 \%)$ \\
\hline
\end{tabular}

\section{Discussion}

Prostate cancer (Gleason score $\geq 6$ ) was detected in $64 \%$ of the lesions biopsied and in $70 \%$ of the patients. All biopsies were representative showing that our novel local reference augmented method is feasible in clinical practice. During MR-TRUS fusion, $88 \%$ of the lesions could be visualized on TRUS alone, allowing targeted biopsies to be optimized using live TRUS guidance. The cancer detection rate increases with increasing tumor size.

The representativeness of the mpMRI suspicious, but negative MRgUSBx was confirmed by follow-up; i.e., none of the seven negative MRgUSBx patients revealed clinically significant pathology. The detection rate for clinically significant cancer $(44 \%)$ was lower than the $65 \%$ shown by Pokorny et al. [11] for MRgMRBx. Three reasons can be pointed out for this difference: (1) different patient population between studies; (2) difference in biopsy technique; and (3) difference in expertise of the radiologist(s). In Pokorny et al. three expert radiologists in consensus evaluated the mpMRI, which in our study was done by the attending prostate radiologist. To investigate the first two points, more research is required comparing MRgUSBx to MRgMRBx with similar patient populations. To investigate the third point, two expert radiologists in consensus reevaluated the original mpMRIs with negative and clinically insignificant MRgUSBx. Table 5 shows that the original mpMRI assessment may indeed have overestimated tumor aggression: $43 \%(6 / 14)$ of the lesions were downgraded during retrospective re-evaluation by experts. This confirms that expertise is important. In case subsequent biopsy reveals no clinically significant cancer in PIRADS 4 and 5 lesions, it is very important to re-evaluate the quality of the mpMRI, the reading, and the subsequent biopsy technique.

The original results of our locally optimized EM-based registration method are well in line with results of other EM-based systems, which have a cancer detection rate between 49 and $69 \%[18,19]$. Clinical studies with other MR-TRUS fusion systems also show similar detection rates $[18,19,25,26]$. However, patient populations differ quite a bit between the different studies, e.g., regarding the amount of patients with a previous negative biopsy or patients that were biopsy naïve. Although our initial results are similar to previously published results, more research is needed to investigate whether local reference augmentation is an actual technical improvement to current MR-TRUS fusion methods.

We know from previous phantom studies that our EMbased registration method has a registration accuracy of about $5 \mathrm{~mm}$ [20]. Yet we were still able to achieve reasonable detection rates in the $0-10 \mathrm{~mm}$ category. The following items played a role. First, we enhanced the EM-based registration technique by locally optimizing fusion through the iterative selection of anatomical landmarks close to the target. Secondly, the number of biopsy cores was increased in case the performing physician was not certain about the biopsy taken. By taking more cores per target, the tumor hit rate increases [17]. Thirdly, during the MRgUSBx we noticed that $88 \%$ of the targets became visible on TRUS images during MR-TRUS fusion. In case the targets become visible on TRUS, these can be more accurately targeted even if the registration is not optimal [27]. The TRUS visibility is often subtle and may very well depend on the quality of the ultrasound images.

The cancer detection rate increases with increasing lesion size, which might be an indication that the smaller lesions are harder to hit with MRgUSBx and might better be biopsied with MRgMRBx. For lesions larger than $10 \mathrm{~mm}$ diameter, our results show a cancer detection rate of $73 \%$, approaching the results of MRgMRBx.

The main limitation of our study is the small number of patients. But sufficient to indicate that MR-TRUS fusion with local reference augmentation is feasible for targeting prostate biopsies. To investigate whether MRgUSBx is a viable alternative to MRgMRBx, a non-inferiority trial setting including more patients is required. Then, similar patient groups can be compared and it can be determined whether MRgUSBx is non-inferior to MRgMRBx.

To summarize, MR-TRUS fusion biopsy using local reference augmentation is feasible. This is especially the case for lesions with an mpMRI maximum diameter of at least $10 \mathrm{~mm}$ or PIRADS 5 lesions. Smaller lesions may still require in-bore MR-guided biopsy. 


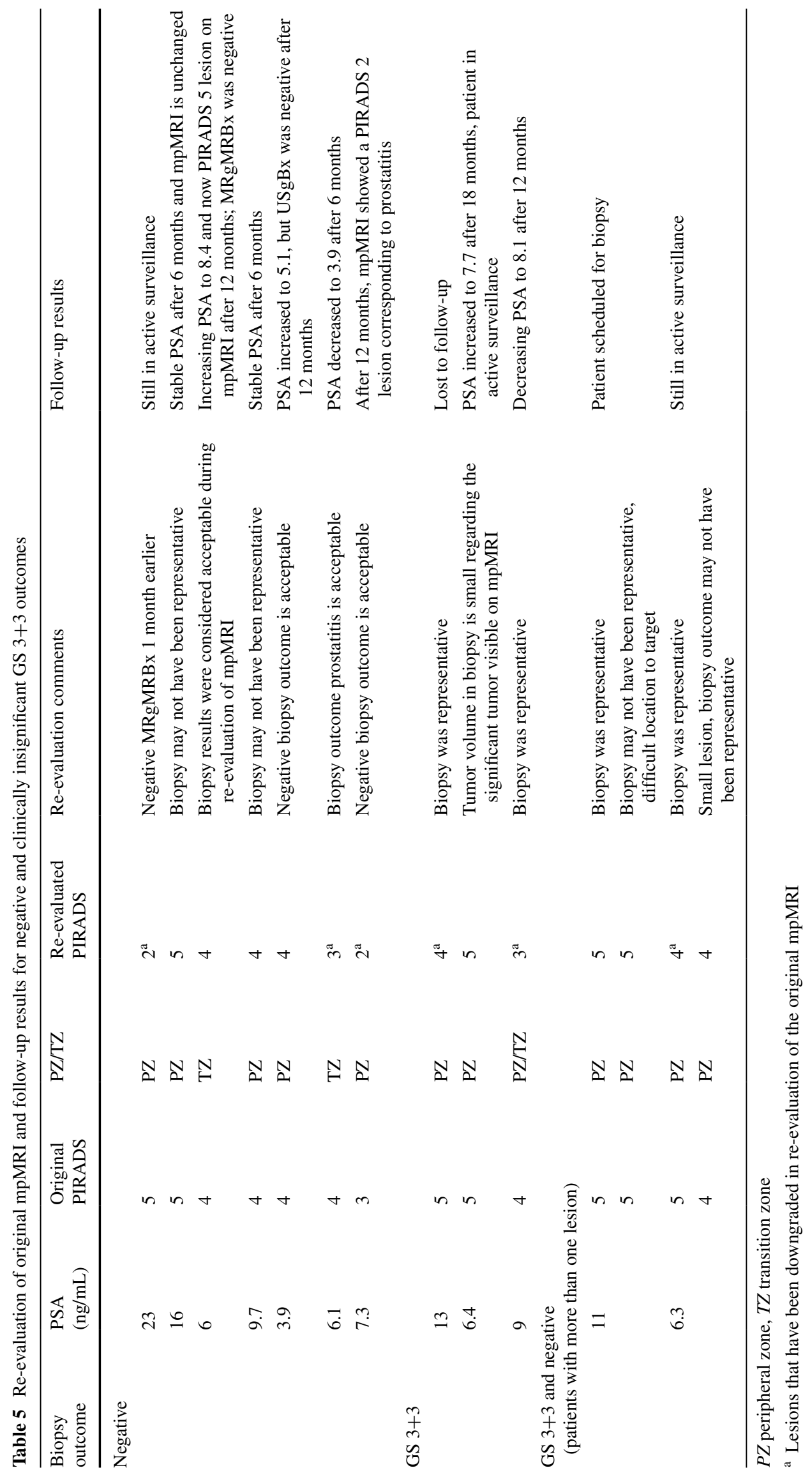


Acknowledgments This study was funded by Grant No. KUN 2007-3971 of the Dutch Cancer Society. H. J. Huisman has received a research grant from Toshiba.

\section{Compliance with ethical standards}

Conflict of interest W. J. M. van de Ven, W. Venderink, J. P. M. Sedelaar, J. Veltman, J. O. Barentsz, J. J. Fütterer, E. B. Cornel declare that they have no conflict of interest.

Ethical approval All procedures performed in studies involving human participants were in accordance with the ethical standards of the institutional and/or national research committee and with the 1964 Helsinki Declaration and its later amendments or comparable ethical standards.

Informed consent Informed consent was obtained from all individual participants included in the study.

Open Access This article is distributed under the terms of the Creative Commons Attribution 4.0 International License (http://creativecommons.org/licenses/by/4.0/), which permits unrestricted use, distribution, and reproduction in any medium, provided you give appropriate credit to the original author(s) and the source, provide a link to the Creative Commons license, and indicate if changes were made.

\section{References}

1. Ukimura O, Coleman JA, de la Taille A, Emberton M, Epstein JI, Freedland SJ, Giannarini G, Kibel AS, Montironi R, Ploussard G, Roobol MJ, Scattoni V, Jones JS (2013) Contemporary role of systematic prostate biopsies: indications, techniques, and implications for patient care. Eur Urol 63:214-230

2. Norberg M, Egevad L, Holmberg L, Sparén P, Norlén BJ, Busch C (1997) The sextant protocol for ultrasound-guided core biopsies of the prostate underestimates the presence of cancer. Urology 50:562-566

3. Roehl KA, Antenor JAV, Catalona WJ (2002) Serial biopsy results in prostate cancer screening study. J Urol 167:2435-2439

4. Terris MK (1999) Sensitivity and specificity of sextant biopsies in the detection of prostate cancer: preliminary report. Urology $54: 486-489$

5. Hambrock T, Hoeks C, Hulsbergen-van de Kaa C, Scheenen T, Fütterer J, Bouwense S, van Oort I, Schröder F, Huisman H, Barentsz J (2012) Prospective assessment of prostate cancer aggressiveness using 3-T diffusion-weighted magnetic resonance imaging-guided biopsies versus a systematic 10-core transrectal ultrasound prostate biopsy cohort. Eur Urol 61:177-184

6. Kvåle R, Møller B, Wahlqvist R, Fossa SD, Berner A, Busch C, Kyrdalen AE, Svindland A, Viset T, Halvorsen OJ (2009) Concordance between Gleason scores of needle biopsies and radical prostatectomy specimens: a population-based study. Br J Urol Int 103:1647-1654

7. Rajinikanth A, Manoharan M, Soloway CT, Civantos FJ, Soloway MS (2008) Trends in Gleason score: concordance between biopsy and prostatectomy over 15 years. Urology 72:177-182

8. Ehdaie B, Vertosick E, Spaliviero M, Giallo-Uvino A, Taur Y, O'Sullivan M, Livingston J, Eastham J, Scardino P, Touijer K (2014) The impact of repeat biopsies on infectious complications in men with prostate cancer on active surveillance. J Urol 191:660-664

9. Kitajima K, Kaji Y, Fukabori Y, Yoshida K, Suganuma N, Sugimura K (2010) Prostate cancer detection with 3 T MRI: comparison of diffusion-weighted imaging and dynamic contrast-enhanced MRI in combination with T2-weighted imaging. J Magn Reson Imaging 31:625-631

10. Tanimoto A, Nakashima J, Kohno H, Shinmoto H, Kuribayashi S (2007) Prostate cancer screening: the clinical value of diffusionweighted imaging and dynamic MR imaging in combination with T2-weighted imaging. J Magn Reson Imaging 25:146-152

11. Pokorny MR, de Rooij M, Duncan E, Schröder FH, Parkinson R, Barentsz JO, Thompson LC (2014) Prospective study of diagnostic accuracy comparing prostate cancer detection by transrectal ultrasound-guided biopsy versus magnetic resonance (MR) imaging with subsequent MR-guided biopsy in men without previous prostate biopsies. Eur Urol 66:22-29

12. Hu Y, Ahmed HU, Taylor Z, Allen C, Emberton M, Hawkes D, Barratt D (2012) MR to ultrasound registration for image-guided prostate interventions. Med Image Anal 16:687-703

13. Kaplan I, Oldenburg NE, Meskell P, Blake M, Church P, Holupka EJ (2002) Real time MRI-ultrasound image guided stereotactic prostate biopsy. Magn Reson Imaging 20:295-299

14. Ukimura O, Hirahara N, Fujihara A, Yamada T, Iwata T, Kamoi K, Okihara K, Ito H, Nishimura T, Miki T (2010) Technique for a hybrid system of real-time transrectal ultrasound with preoperative magnetic resonance imaging in the guidance of targeted prostate biopsy. Int J Urol 17:890-893

15. van de Ven WJM, Hulsbergen-van de Kaa CA, Hambrock T, Barentsz JO, Huisman HJ (2013) Simulated required accuracy of image registration tools for targeting high-grade cancer components with prostate biopsies. Eur Radiol 23:1401-1407

16. Singh AK, Kruecker J, Xu S, Glossop N, Guion P, Ullman K, Choyke PL, Wood BJ (2008) Initial clinical experience with realtime transrectal ultrasonography-magnetic resonance imaging fusion-guided prostate biopsy. Br J Urol Int 101:841-845

17. Martin PR, Cool DW, Romagnoli C, Fenster A, Ward AD (2014) Magnetic resonance imaging-targeted, 3D transrectal ultrasoundguided fusion biopsy for prostate cancer: quantifying the impact of needle delivery error on diagnosis. Med Phys 41:073504

18. Delongchamps NB, Peyromaure M, Schull A, Beuvon F, Bouazza N, Flam T, Zerbib M, Muradyan N, Legman P, Cornud F (2013) Prebiopsy magnetic resonance imaging and prostate cancer detection: comparison of random and targeted biopsies. J Urol 189:493-499

19. Puech P, Rouvière $O$, Renard-Penna R, Villers A, Devos P, Colombel M, Bitker M-O, Leroy X, Mège-Lechevallier F, Comperat E, Ouzzane A, Lemaitre L (2013) Prostate cancer diagnosis: multiparametric MR-targeted biopsy with cognitive and transrectal US-MR fusion guidance versus systematic biopsyprospective multicenter study. Radiology 268:461-469

20. van de Ven WJM, Rinsma S, Karssemeijer N, Barentsz JO, Huisman HJ (2014) Electro-magnetic tracker-based fusion for image-guided TRUS prostate biopsy. In: European congress of radiology

21. van de Ven WJM, Sedelaar JPM, Fütterer JJ, Huisman HJ (2014) Focally targeted magnetic resonance imaging guided transrectal ultrasound biopsy of the prostate with an ultrasound machine that has electromagnetic tracking fusion. In: Annual meeting of the radiological society of North America

22. Barentsz JO, Richenberg J, Clements R, Choyke P, Verma S, Villeirs G, Rouvière O, Logager V, Fütterer JJ, European Society of Urogenital Radiology (2012) ESUR prostate MR guidelines 2012. Eur Radiol 22:746-757

23. Huisman H, Vos P (2010) MRCAD for daily clinical analysis of prostate MR. Kitware Source 13:14-15

24. Epstein JI, Allsbrook WC, Amin MB, Egevad LL, Grading ISUP, ISUP Grading Committee (2005) The 2005 International Society of Urological Pathology (ISUP) consensus conference 
on Gleason grading of prostatic carcinoma. Am J Surg Pathol 29:1228-1242

25. Siddiqui MM, Rais-Bahrami S, Truong H, Stamatakis L, Vourganti S, Nix J, Hoang AN, Walton-Diaz A, Shuch B, Weintraub M, Kruecker J, Amalou H, Turkbey B, Merino MJ, Choyke PL, Wood BJ, Pinto PA (2013) Magnetic resonance imaging/ultrasound-fusion biopsy significantly upgrades prostate cancer versus systematic 12-core transrectal ultrasound biopsy. Eur Urol 64:713-719

26. Sonn GA, Chang E, Natarajan S, Margolis DJ, Macairan M, Lieu P, Huang J, Dorey FJ, Reiter RE, Marks LS (2013) Value of targeted prostate biopsy using magnetic resonance-ultrasound fusion in men with prior negative biopsy and elevated prostatespecific antigen. Eur Urol 65:809-815

27. Ukimura O, Marien A, Palmer S, Villers A, Aron M, de Castro Abreu AL, Leslie S, Shoji S, Matsugasumi T, Gross M, Dasgupta P, Gill IS (2015) Trans-rectal ultrasound visibility of prostate lesions identified by magnetic resonance imaging increases accuracy of image-fusion targeted biopsies. World J Urol 33(11):1669-1676 